\title{
Equivalent equations of motion for gravity and entropy
}

\author{
Bartłomiej Czech, ${ }^{a}$ Lampros Lamprou, ${ }^{a}$ Samuel McCandlish, ${ }^{a}$ Benjamin Mosk $^{a}$ and \\ James Sully ${ }^{b}$ \\ a Physics Department, Stanford Institute for Theoretical Physics, Stanford University, \\ 382 Via Pueblo Mall, Stanford, CA 94305, U.S.A. \\ ${ }^{b}$ Theory Group, SLAC National Accelerator Laboratory, \\ 2575 Sand Hill Road, Menlo Park, CA 94025, U.S.A. \\ E-mail: czech@stanford.edu, llamprou@stanford.edu, \\ smccandlish@stanford.edu, bmosk1@stanford.edu, \\ jsully@slac.stanford.edu
}

ABSTRACT: We demonstrate an equivalence between the wave equation obeyed by the entanglement entropy of CFT subregions and the linearized bulk Einstein equation in Anti-de Sitter space. In doing so, we make use of the formalism of kinematic space [1] and fields on this space, introduced in [2]. We show that the gravitational dynamics are equivalent to a gauge invariant wave-equation on kinematic space and that this equation arises in natural correspondence to the conformal Casimir equation in the CFT.

KEYwords: AdS-CFT Correspondence, Conformal Field Theory

ARXIV EPRINT: 1608.06282 


\section{Contents}

1 Introduction 1

$\begin{array}{llr}2 & \text { Scalar kinematic dictionary } & 2\end{array}$

3 Tensor Radon transforms and Einstein's equations 5

4 Modular Hamiltonian and the tensor kinematic dictionary 8

$\begin{array}{lll}5 & \text { Discussion } & 10\end{array}$

A Tensor Radon transforms

$\begin{array}{ll}\text { A.1 Tensor intertwinement } & 12\end{array}$

$\begin{array}{lll}\text { A.2 Einstein equations from intertwinement } & 14\end{array}$

$\begin{array}{ll}\text { B Wedge integral relations } & 15\end{array}$

$\begin{array}{ll}\text { C Modular Hamiltonian as an OPE block } & 17\end{array}$

\section{Introduction}

The recent direct detection of gravitational waves [3] adds to an impressive list of observational tests of general relativity, the theory that describes long-distance dynamics of spacetime. At the quantum level, however, the fate of spacetime requires a more careful assessment; indeed, it is not even clear what the fundamental degrees of freedom in a theory of quantum gravity are. The holographic principle $[4,5]$ suggests that these degrees of freedom should in fact be nonlocal.

This notion is made explicit in the AdS/CFT duality, an equivalence between $d$-dimensional conformal field theories (CFTs) and $(d+1)$-dimensional gravitational systems with Anti de Sitter (AdS) asymptotics. In particular, the proposal of Ryu and Takayangi (RT) $[6,7]$ relates the entanglement entropy $S$ of a CFT region $B$ to the area of a bulk extremal surface $\tilde{B}$,

$$
S(B)=\min _{\partial \tilde{B}=\partial B} \frac{\operatorname{area}(\tilde{B})}{4 G_{N}} .
$$

Developments over the past few years [8-12] have shown that the dynamics of this nonlocal CFT quantity is closely related to the bulk Einstein equation.

In recent work [2] we demonstrated an extension of the RT proposal to other bulk scalar fields. We introduced the OPE block, the contribution to the CFT operator product expansion (OPE) from a single conformal family, and equated it to the Radon transform, 
the integral of a bulk scalar field over a minimal surface. Both of these objects obey an equation of motion in kinematic space, a space which geometrizes the set of bulk surfaces of a given dimension. We showed that this kinematic equation of motion emerges directly from the bulk Klein-Gordon equation.

In this paper, we will demonstrate the relationship between the Ryu-Takayanagi proposal and our results on kinematic space. Specifically, we will show that the wave equations obeyed by a perturbation to entanglement entropy [11-15] correspond directly to the linearized bulk Einstein equation with matter via an intertwining relation of the Radon transform:

$$
\begin{aligned}
& \left(\square_{\mathcal{K}}+2 d\right) \delta S=0 \quad \leftrightarrow \quad \text { Einstein equations } \\
& \left(\square_{\mathrm{dS}}+d\right) \delta S=0 \quad \leftrightarrow \quad \text { Hamiltonian constrant }
\end{aligned}
$$

where the Laplacians $\square_{\mathcal{K}}$ and $\square_{\text {dS }}$ on kinematic space arise from conformal Casimir equations. This clarifies and connects the results of [8-12].

Before stating our result explicitly, we begin by outlining the results of [2] on kinematic space, the OPE block, and the Radon transform.

\section{Scalar kinematic dictionary}

The kinematic dictionary, introduced in [2] and recently also explored in [16], connects Radon transforms of AdS-fields with OPE-blocks in the dual CFT. Here we outline the basic formalism.

Radon transform. The Radon transform is a map from functions $f(x)$ on some manifold to functions on the space of $n$-dimensional totally geodesic submanifolds $\tilde{B}$. It is defined via the integral transform

$$
R[f](\tilde{B})=\int_{\tilde{B}} d A f
$$

where $d A$ is the induced area element on the surface $\tilde{B}$. Though [2] also considered the case $n=1$ of geodesics, we will focus here on the case $n=d-1$ of codimension- 2 minimal surfaces in $\mathrm{AdS}_{d+1}$.

It is useful to define an auxiliary space $\mathcal{K}$, which we call kinematic space, to organize information about bulk surfaces. A point in $\mathcal{K}$ denotes equivalently any of the following:

- a particular bulk minimal surface, $\tilde{B}$,

- the boundary sphere where that surface ends, $B$,

- the two timelike separated boundary points $x_{1}, x_{2}$ at the tips of the causal development of this sphere (see figure 2), 12 .

The points of $\mathcal{K}$ are most conveniently parameterized by the two points $\left(x_{1}, x_{2}\right)$. When the context is clear, we will often denote any of the above three objects by the pair $\left(x_{1}, x_{2}\right)$. 


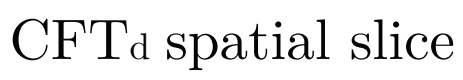

$\mathrm{dS}(1, \mathrm{~d}-1)$
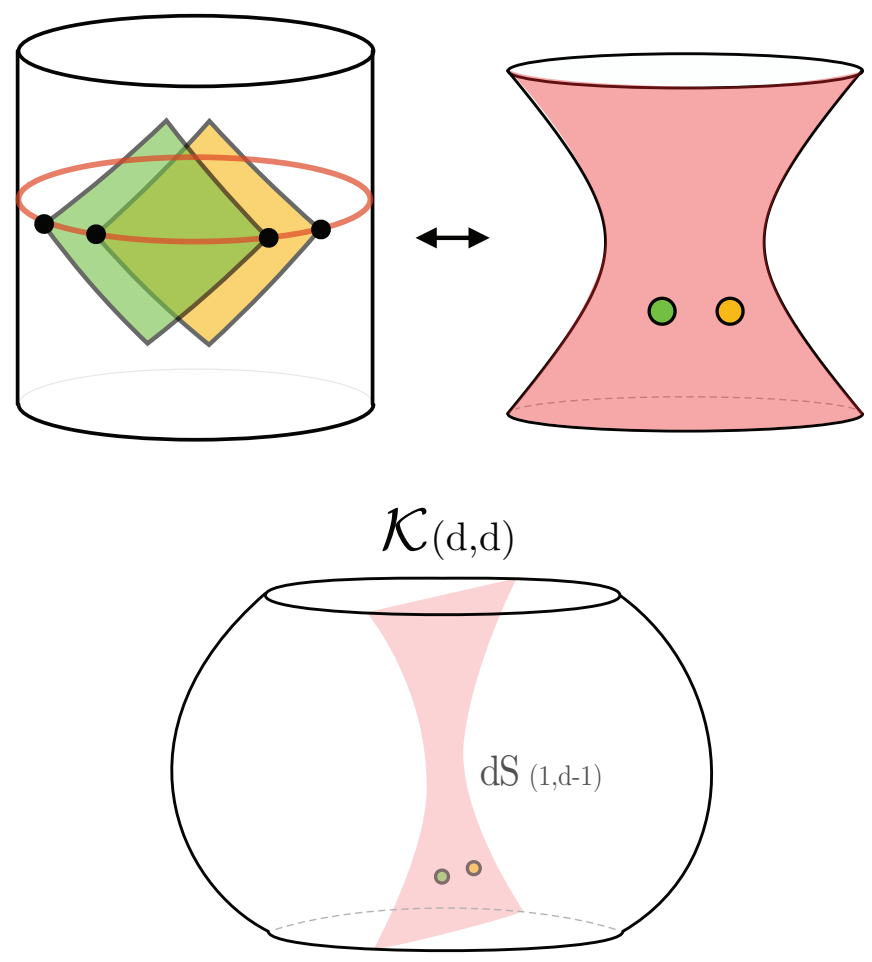

Figure 1. The kinematic space for spherical regions that lie on a single time slice is given by $d$-dimensional Lorentzian de Sitter space. The de Sitter space is a corresponding slice of the larger kinematic space for all (boosted) spherical regions, which is $2 d$-dimensional with signature $(d, d)$.

The conformal group $\mathrm{SO}(d, 2)$ then endows kinematic space with a metric structure of $(d, d)$ signature (see $[2])$,

$$
d s^{2}=\frac{I_{\mu \nu}\left(x_{1}-x_{2}\right)}{\left(\frac{x_{1}-x_{2}}{2}\right)^{2}} d x_{1}^{\mu} d x_{2}^{\nu}
$$

where $I_{\mu \nu}(x)=\eta_{\mu \nu}-2 \frac{x_{\mu} x_{\nu}}{x^{2}}$ is the CFT inversion tensor.

It will also be useful to note that if we consider only spheres living in a particular equal-time slice of $\operatorname{AdS}_{d+1}$, preserved by an $\mathrm{SO}(d, 1)$ subgroup, the corresponding slice of $\mathcal{K}$ has the structure of a $d$-dimensional de Sitter (dS) space $[1,2,14,17]$ (see figure 1). There is one such dS slice for each time slice of AdS. For instance, if we consider the $t=0$ slice of AdS and parameterize the spheres it contains by their radius $R$ and center $\vec{x}$, the induced metric on this slice is given by

$$
d s^{2}=\frac{-d R^{2}+d \vec{x}^{2}}{R^{2}} .
$$

A particularly useful feature of the kinematic space $\mathcal{K}$ is that the domain of the CFT, including the time direction, appears as a spacelike surface at $x_{1}=x_{2}$. This allows us to 


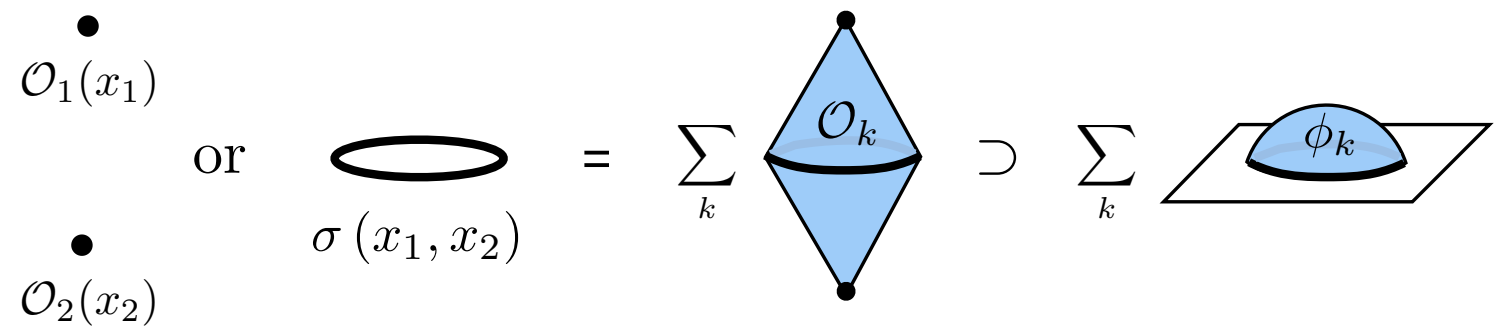

Figure 2. A point in kinematic space labels two timelike-separated boundary points, $x_{1}, x_{2}$, or equivalently a codimension- 2 sphere formed by their causal domain. Operators $\sigma\left(x_{1}, x_{2}\right)$ localized on the sphere have an OPE expansion in terms of OPE blocks for primaries $\mathcal{O}_{k}$, where contributions can be identified as bulk surface operators $\phi_{k}$. Figure from [2].

impose boundary conditions and choose a causal propagator in the usual way when solving wave equations in kinematic space.

OPE-blocks. We now introduce a CFT object whose domain is also $\mathcal{K}$. Recall that in a CFT, the product of two identical scalar local operators can be expanded in terms of the global primary operators of the theory as

$$
\frac{\mathcal{O}\left(x_{1}\right) \mathcal{O}\left(x_{2}\right)}{\left\langle\mathcal{O}\left(x_{1}\right) \mathcal{O}\left(x_{2}\right)\right\rangle}=\sum_{k} C_{\mathcal{O O} k} \underbrace{\left|x_{12}\right|^{\Delta_{k}}\left(1+b_{1} x_{12}^{\mu} \partial_{\mu}+\cdots\right) \mathcal{O}_{k}\left(x_{2}\right)}_{\mathcal{B}_{k}\left(x_{1}, x_{2}\right)}
$$

where $x_{12}^{\mu}=x_{1}^{\mu}-x_{2}^{\mu}$. The coefficients $C_{\mathcal{O O} k}$ are known as the OPE coefficients and are theory-dependent, while the coefficients $b_{i}$ depend only on the scaling dimension $\Delta_{k}$ of the primary operator $\mathcal{O}_{k}$. We have grouped the contributions from a single primary to the OPE into an object $\mathcal{B}_{k}\left(x_{1}, x_{2}\right)$, which we call the OPE-block. In [2], we showed that operators $\sigma\left(x_{1}, x_{2}\right)$ localized on a CFT sphere can be expanded in terms of the same OPE blocks as

$$
\frac{\sigma\left(x_{1}, x_{2}\right)}{\left\langle\sigma\left(x_{1}, x_{2}\right)\right\rangle}=\sum_{k} C_{\sigma k} \mathcal{B}_{k}\left(x_{1}, x_{2}\right)
$$

where $C_{\sigma k}$ are theory-dependent "OPE" coefficients.

The OPE-block also has a compact integral expression [2] over the causal diamond formed by the points $x_{1}, x_{2}$. For a scalar, this is just:

$$
\mathcal{B}_{k}\left(x_{1}, x_{2}\right)=N_{k} \int_{\downarrow_{12}} d^{d} x_{3}\left(\frac{x_{13} x_{23}}{x_{12}}\right)^{\Delta_{k}-d} \mathcal{O}_{k}\left(x_{3}\right) .
$$

OPE-blocks are useful as CFT objects in their own right; however, they become even more powerful in the presence of an AdS dual, as we describe next.

Kinematic dictionary. At leading order in the $N \rightarrow \infty$ limit, the OPE-block $\mathcal{B}_{k}\left(x_{1}, x_{2}\right)$ and the Radon transform $R\left[\phi_{k}\right]\left(x_{1}, x_{2}\right)$ of the dual AdS field are directly related:

$$
\mathcal{B}_{k}\left(x_{1}, x_{2}\right)=\frac{1}{c_{\Delta_{k}}} R\left[\phi_{k}\right]\left(x_{1}, x_{2}\right)
$$


where $c_{\Delta}$ is a constant depending only on the dimension $d$ and the scaling dimension $\Delta_{k}$. To prove this, in [2] we showed that both sides obey the same equation of motion with the same boundary conditions:

$$
\begin{array}{rc}
\left(\square_{\mathcal{K}}+m_{k}^{2}\right) \mathcal{B}_{k}=0 & \left(\square_{\mathcal{K}}+m_{k}^{2}\right) R\left[\phi_{k}\right]=0 \\
\mathcal{B}_{k} \sim\left|x_{12}\right|^{\Delta_{k}} \mathcal{O}_{k} & R\left[\phi_{k}\right] \sim c_{\Delta_{k}}\left|x_{12}\right|^{\Delta_{k}} \mathcal{O}_{k} .
\end{array}
$$

The OPE block equation of motion comes from a conformal Casimir equation,

$$
\left[L^{2}, \mathcal{B}_{k}\right]=\square_{\mathcal{K}} \mathcal{B}_{k}=C_{k} \mathcal{B}_{k}
$$

where $L^{2}$ is the Casimir element of the conformal group $\mathrm{SO}(d, 2)$, with eigenvalue

$$
C_{k}^{\mathrm{SO}(d, 2)}=-\Delta(\Delta-d)-\ell(\ell+d-2)=-m_{k}^{2} .
$$

The Casimir element is represented on kinematic space scalar fields by the Laplacian $\square_{\mathcal{K}}$, yielding the equation of motion. The boundary condition as $x_{1} \rightarrow x_{2}$ comes from inspecting the definition (2.4).

To find the equation of motion for the Radon transform we use an intertwining property:

$$
\square_{\mathcal{K}} R[\phi]=-R\left[\square_{\mathrm{AdS}} \phi\right] .
$$

Together with the Klein-Gordon equation, this implies an equation of motion for the Radon transform:

$$
\square_{\mathrm{AdS}} \phi_{k}=m_{k}^{2} \phi_{k} \quad \Longrightarrow \quad \square_{\mathcal{K}} R\left[\phi_{k}\right]=-m_{k}^{2} R\left[\phi_{k}\right] .
$$

The boundary condition then comes from the AdS/CFT dictionary $\phi_{k}(x, z) \rightarrow z^{\Delta_{k}} \mathcal{O}_{k}(x)$.

Since kinematic space has signature $(d, d)$, an additional $d-1$ equations are required to fix a solution uniquely. These take the form of constraint equations, explained in detail in $[2,16]$. These equations of motion together with the boundary conditions establish the validity of (2.7).

In the remainder of this paper, we will use the same techniques to extend the kinematic dictionary to the CFT stress tensor, which is dual to the bulk metric perturbation. We will extend this dictionary to first subleading order in the $1 / N$ expansion, finding that the correction is precisely that found in [18]. This will allow us to prove an equivalence between the linearized Einstein's equations in the bulk and a simple equation satisfied by the stress tensor OPE-block, which we show is equal to the modular Hamiltonian.

\section{Tensor Radon transforms and Einstein's equations}

In this section, we will show that the linearized Einstein equations are equivalent to a set of equations obeyed by the fluctuation in the area of the minimal surfaces. We will do this in a similar way as in eq. (2.12), by using an intertwining relation. The goal will be to find an analog of the field equation and boundary conditions of eq. (2.8); we will then match to CFT quantities in the following section. 
Since the bulk field of interest, the metric perturbation $\delta g_{\mu \nu}$, is a tensor, we must first introduce a tensor analog of the Radon transform eq. (2.1). For a symmetric 2-tensor field $s_{\mu \nu}$, we define the longitudinal and transverse Radon transforms, denoted $R_{\|}$and $R_{\perp}$ respectively, as

$$
\begin{aligned}
& R_{\|}\left[s_{\mu \nu}\right]\left(x_{1}, x_{2}\right)=\int_{\tilde{B}_{12}} d A h^{\mu \nu} s_{\mu \nu} \\
& R_{\perp}\left[s_{\mu \nu}\right]\left(x_{1}, x_{2}\right)=\int_{\tilde{B}_{12}} d A\left(g^{\mu \nu}-h^{\mu \nu}\right) s_{\mu \nu} .
\end{aligned}
$$

Here, $h_{\mu \nu}$ denotes the induced metric on the surface $\tilde{B}_{12}$. As before, these transforms output a scalar function on kinematic space; we write indices on the left side only to indicate that the input is a tensor.

We now note some useful identities for the tensor Radon transform. First, note that the sum of the two tensor transforms is just a scalar Radon transform of trs. This implies that the two are related by a trace-reversal of the input tensor,

$$
\begin{aligned}
R_{\|}\left[s_{\mu \nu}\right] & =-R_{\perp}\left[s_{\mu \nu}-\frac{1}{2} g_{\mu \nu} \operatorname{tr} s\right] \\
R_{\perp}\left[s_{\mu \nu}\right] & =-R_{\|}\left[s_{\mu \nu}-\frac{1}{d-1} g_{\mu \nu} \operatorname{tr} s\right] .
\end{aligned}
$$

Before we continue, let us pause to note a striking simplification of the full nonlinear Einstein equation when written in terms of tensor Radon transforms. The Einstein equation takes the form

$$
R_{\mu \nu}-\frac{1}{2} g_{\mu \nu} R=8 \pi G_{N} T_{\mu \nu}
$$

where we have defined $T_{\mu \nu}$ to include the cosmological constant term. If we apply the transverse transform $R_{\perp}$ to both sides, using the identity (3.2), we obtain

$$
\frac{1}{4 G_{N}} R_{\|}\left[R_{\mu \nu}\right]+2 \pi R_{\perp}\left[T_{\mu \nu}\right]=0 .
$$

This remarkable simplification occurs only when we integrate over a codimension-2 surface, due to the appearance of the coefficient $\frac{1}{2}$ in the Einstein tensor. ${ }^{1}$

Let us now consider the linearized version of eq. (3.4). Setting $T_{\mu \nu}=-\frac{\Lambda}{8 \pi G_{N}} g_{\mu \nu}+\delta T_{\mu \nu}$, with $\Lambda=-\frac{1}{2} d(d-1)$ for $\operatorname{AdS}_{d+1}$, we have

$$
\frac{1}{4 G_{N}} R_{\|}\left[\delta R_{\mu \nu}\right]=-2 \pi R_{\perp}\left[\delta T_{\mu \nu}\right]-\frac{2 d}{4 G_{N}} \delta A
$$

where $\delta A$ is the first order change in the area of the surface of integration. In terms of the tensor Radon transform, the area perturbation can be written as

$$
\delta A\left(x_{1}, x_{2}\right)=\frac{1}{2} \int_{\tilde{B}_{12}} h^{\mu \nu} \delta g_{\mu \nu} d A=\frac{1}{2} R_{\|} \delta g .
$$

\footnotetext{
${ }^{1}$ However, note for a dimension-2 (rather than codimension-2) surface, the longitudinal transform of the Einstein equations yields the equation

$$
R_{\perp}^{(2)}\left[R_{\mu \nu}\right]+R_{\|}^{(2)}\left[T_{\mu \nu}\right]=0 .
$$


We would like to specialize to minimal surfaces in $\mathrm{AdS}_{d+1}$, and recast the linearized equation (3.6) as an equation of motion in kinematic space. To do this, we make use of an intertwining relation analogous to $(2.11)$,

$$
\square_{K} R_{\|}\left[s_{\mu \nu}\right]=-R_{\|}\left[\left(\nabla^{2}+2(d+1)\right) s_{\mu \nu}-2 g_{\mu \nu} \operatorname{tr} s\right],
$$

where $\nabla^{2}=\nabla_{\alpha} \nabla^{\alpha}$ denotes the covariant Laplacian. The right side of this equation is given by the action of the casimir $L_{\mathrm{SO}(d, 2)}^{2}$ on $s_{\mu \nu}$, as shown in appendix A.1. In fact, for the case of $\delta g_{\mu \nu}$, eq. (3.8) can be rewritten as

$$
\square_{K} \delta A=R_{\|}\left[\delta R_{\mu \nu}\right],
$$

where $\delta R_{\mu \nu}$ is the variation of the Ricci tensor due to the variation $\delta g_{\mu \nu}$ in the metric; this is shown explicitly in appendix A.2. Together with (3.6), this implies the equation of motion

$$
\left(\square_{K}+2 d\right) \frac{\delta A}{4 G_{N}}=-2 \pi R_{\perp}[\delta T] .
$$

We have thus shown that the area perturbation $\delta A$ obeys an equation of motion in kinematic space as a consequence of the linearized Einstein equation about AdS.

To show complete equivalence between the kinematic equation of motion and Einstein equations, it remains only to show that the tensor Radon transform is invertible (up to diffeomorphisms). Unfortunately, while reasonable, we are not aware of a proof of this fact in the literature, and our statement of equivalence must carry a technical asterisk awaiting further input from the mathematical community.

To avoid the technical problem in the preceding paragraph, we will now prove an additional equation of motion for the area perturbation, but this time restricting ourselves to surfaces on a time slice of AdS; this corresponds to a particular de Sitter slice of kinematic space. Using the same techniques as above, we prove in appendix A.2 that

$$
\left(\square_{\mathrm{dS}}+d\right) \delta A=R\left[\delta\left(R_{\mu \nu}-\frac{1}{2} R g_{\mu \nu}\right) \hat{t}^{\mu} \hat{t}^{\nu}\right] .
$$

The right hand side is a $t t$ component of the Einstein tensor; hence, using the Einstein equation, we find

$$
\left(\square_{\mathrm{dS}}+d\right) \frac{\delta A}{4 G_{N}}=2 \pi R\left[\delta T_{00}\right] .
$$

Here, $T_{00}$ denotes the energy density relative to the particular AdS time slice we are considering; there is a separate de Sitter equation for each time slice. Hence, the Hamiltonian constraint of the Einstein equation implies a de Sitter equation of motion for the area perturbation. In thise case, since the scalar Radon transform is known to be injective [19], eq. (3.12) is equivalent to the Hamiltonian constraint on a time slice, and the collection of de Sitter equations for every slice is equivalent to the full linearized Einstein equation, because knowing $E_{00}=T_{00}$ for every choice of $\hat{t}$ implies $E_{\mu \nu}=T_{\mu \nu}$.

To complete the description of the Cauchy problem for the area perturbation $\delta A$ in kinematic space, we must fix boundary conditions. This can be done using the extrapolate dictionary for the metric perturbation [20-22], and was shown in [9] to be

$$
\delta A\left(x_{0}, R\right) \underset{R \rightarrow 0}{\sim} R^{d} T_{00}\left(x_{0}\right) \frac{8 \pi G_{N} \Omega_{d-2}}{d^{2}-1} .
$$


Now that we have formulated the Cauchy problem for the area perturbation in the form of eq. (3.13) along with either (3.10) or (3.12), we can proceed to match with CFT variables.

\section{Modular Hamiltonian and the tensor kinematic dictionary}

In this section we use the Radon-transformed Einstein equations we have just derived to give a novel derivation of the quantum-corrected Ryu-Takayanagi formula.

To begin, we take a moment to review the entanglement first law and the form of the vacuum modular Hamiltonian. Given a quantum mechanical system in a certain state $|\psi\rangle$, the state of a subsystem $B$ is described by the reduced density matrix $\rho_{B}$, obtained from $|\psi\rangle\langle\psi|$ by tracing over the degrees of freedom of the complement $B^{c}$. For such a subsystem, the entanglement entropy is defined as:

$$
S=-\operatorname{tr} \rho_{B} \log \rho_{B}
$$

The modular Hamiltonian $H_{\text {mod }}$ of the state $\rho_{B}$ is then defined implicitly by

$$
\rho_{B}=\frac{e^{-H_{\mathrm{mod}}}}{\operatorname{tr}\left(e^{-H_{\mathrm{mod}}}\right)} .
$$

Using this expression, the change in the entanglement entropy of $A$ due to a small perturbation of the state can be compactly expressed as:

$$
\delta S=\delta\left\langle H_{\mathrm{mod}}\right\rangle
$$

This equation is known as the first law of entanglement entropy.

When $B$ is a ball of radius $R$ in the vacuum state of a CFT, the modular Hamiltonian can be written as

$$
H_{\text {mod }}=2 \pi \int_{B} d^{d-1} x \frac{R^{2}-\left(x-x_{0}\right)^{2}}{2 R} T_{00}(x),
$$

where $T_{00}$ is the energy density in the CFT. The form of the vacuum modular Hamiltonian was computed in [23]. The fact that $H_{\text {mod }}$ is an OPE block was pointed out in [2]; in appendix $\mathrm{C}$ we give the details for general dimension.

We can now make contact with the equations of motion (3.10), (3.12). It was pointed out by [14] that the vacuum modular Hamiltonian, when viewed as a field on kinematic space, obeys a de Sitter wave equation

$$
\left(\square_{\mathrm{dS}}+d\right) H_{\mathrm{mod}}=0 .
$$

It in fact obeys a separate equation for each CFT time slice, each of which has a corresponding de Sitter slice of the full kinematic space $\mathcal{K}$. To see this, note that the $\mathrm{SO}(d, 1)$ subgroup of the conformal group that preserves a time slice has the Casimir

$$
C_{\mathrm{SO}(d, 1)}=-\Delta(\Delta-d+1)-\ell(\ell+d-3) .
$$

Since $T_{00}(x)$ transforms as a scalar of dimension $\Delta=d$ under this subgroup, its Casimir eigenvalue is $-d$. Then $H_{\text {mod }}$, being an integral of $T_{00}$, satisfies $\left[L_{\mathrm{SO}(d, 1)}^{2}, H_{\mathrm{mod}}\right]=-d H_{\mathrm{mod}}$. 
Since $H_{\text {mod }}$ transforms as a scalar field on kinematic space, the Casimir $L_{\mathrm{SO}(d, 1)}^{2}$ is represented by the Laplacian $\square_{\mathrm{dS}}$, yielding the equation (4.5). It follows similarly that $H_{\text {mod }}$ obeys an equation of motion on the full kinematic space,

$$
\left(\square_{\mathcal{K}}+2 d\right) H_{\text {mod }}=0,
$$

with eigenvalue $2 d$ coming from eq. (2.10) [2].

Having written an equation of motion for $H_{\text {mod }}$, we would now like to check the boundary conditions in kinematic space for $H_{\text {mod }}$. Taking the limit $R \rightarrow 0$ of eq. (4.4), we find

$$
H_{\text {mod }}\left(x_{0}, R\right) \underset{R \rightarrow 0}{\sim} R^{d} T_{00}\left(x_{0}\right) \frac{2 \pi \Omega_{d-2}}{d^{2}-1} .
$$

We can now compare directly with the results of section 3. First, let us consider the leading order in $1 / N$ behavior, for which $\delta T_{\mu \nu}=0$. In that case, eqs. (3.12) and (4.5) match, and the boundary conditions (3.13) and (4.8) differ only by a constant $4 G_{N}$. This gives us the leading-order kinematic dictionary:

$$
H_{\text {mod }}=\frac{\delta A}{4 G_{N}}+O\left(N^{0}\right) .
$$

Of course, this is just the linearized Ryu-Takayanagi formula (1.1).

To find the $O\left(N^{0}\right)$ correction to the dictionary, we must find an object $X$ which satisfies

$$
\left(\square_{d S}+d\right) X=-2 \pi R\left[\delta T_{00}\right] .
$$

Using eq. (3.12), this will the guarantee that $\frac{\delta A}{4 G_{N}}+X$ satisfies the same EOM as $H_{\text {mod }}$, eq. (4.5). The solution can be written as

$$
X\left(x_{1}, x_{2}\right)=-2 \pi \int_{\triangle} G_{\mathrm{dS}}^{\mathrm{ret}}\left(x_{1}, x_{2} ; x_{3}, x_{4}\right) R\left[\delta T_{00}\right]\left(x_{3}, x_{4}\right) d V
$$

where the integration region is the past light cone of $\left(x_{1}, x_{2}\right)$, and where $G_{\mathrm{dS}}$ is a bulk-tobulk kinematic space causal propagator. The result is ${ }^{2}$

$$
X=2 \pi \int_{\Sigma} \delta T_{\mu \nu} \xi^{\mu} d \Sigma^{\nu}
$$

where $\xi^{\mu}$ is the Killing vector corresponding to modular flow [16], and where $d \Sigma^{\nu}$ is the timelike unit normal vector to $\Sigma$ (see figure 3 ).

We immediately recognize that $X$ is none other than $H_{\text {bulk }}+\hat{S}_{\text {Wald-like, the bulk vacuum }}$ modular Hamiltonian for a Rindler wedge. (In appendix B, we show via an intertwining relation that $H_{\text {bulk }}$ indeed satisfies eq. (4.10).) We thus arrive at the corrected kinematic dictionary,

$$
H_{\text {mod }}=\frac{\delta A}{4 G_{N}}+H_{\text {bulk }}+\hat{S}_{\text {Wald-like }}
$$

which reproduces the FLM correction to the Ryu-Takayanagi formula [18] as well as the JLMS formula [24]. We hence recognize the Ryu-Takayanagi formula arises as a special case of our more general kinematic dictionary.

\footnotetext{
${ }^{2}$ Note that $\delta T_{\mu \nu}$ refers to the gravitational stress tensor, which appears on the right-hand side of the Einstein equation, rather than the canonical stress tensor [18, 24].
} 


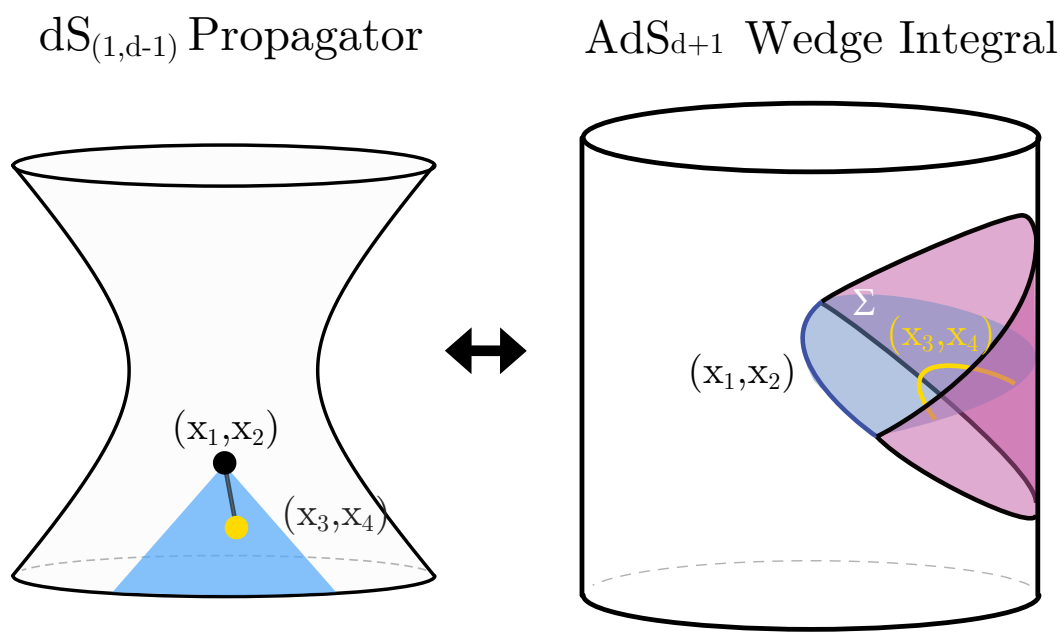

Figure 3. The retarded de Sitter propagator corresponds to an integral over all the geodesics that lie on the $t=0$ slice of the causal wedge. This integral reconstructs the bulk modular Hamiltonian, which is also an integral over the same spatial slice.

\section{Discussion}

We have given a simple and elegant demonstration of the equivalence of the Einstein equations and the quantum-corrected Ryu-Takayanagi formula for linearized perturbations about the vacuum. In doing so, we exploited the fact that both the bulk Einstein equations and the boundary modular Hamiltonian obey simple dynamical equations in an auxiliary kinematic space.

The derivation of the quantum corrected Ryu-Takayanagi formula from the Einstein equations was already described in [18]. While the previous work is more general, the bulk quantum contribution depends on a generally non-local and unknown modular Hamiltonian. Our approach, on the other hand, makes more explicit how quantum corrections arise from bulk interactions. We hope that these techniques will prove insightful when extended away from simple regions of the vacuum state.

This paper was also primarily focused on demonstrating how the bulk Einstein equations imply the Ryu-Takayanagi formula, but our work equally leads to the reverse statement. This is most mathematically rigorous when we make use of the kinematic space for a single-time slice and the scalar Radon transform to derive the $t t$-component of the Einstein equations (all components then follow by appropriate boosts, as in [8]). Here, we can derive the local bulk EOM because the scalar Radon transform on Hyperbolic space has been proven to be invertible [19]. However, to exploit the full kinematic space and directly derive any component of the Einstein equations, we must invert the tensor Radon transform. While the invertibility of these transforms (up to diffeomorphism) is well-motivated, we are not aware of a mathematical proof. Thus, a stickler for rigor will conclude that only an integrated version of the Einstien equations has been derived by this second approach. 
As the gravitational equations of motion intertwine to become a kinematic equation of motion which is fixed by conformal invariance, it may be confusing to wonder what happens to the gravitational equations of motion in a generalized theory of gravity. However, the particular gravitational equation of motion is determined not by the fixed kinematic EOM, but by the choice of entropy functional. In particular, it was shown in [8-10] using the Wald-Iyer formalism [25] that the entanglement first law 4.3 can be written for perturbations of the vacuum as

$$
0=\delta S-\delta\left\langle H_{\mathrm{mod}}\right\rangle=\int_{\Sigma}\left[\frac{1}{4 G_{N}} \delta E_{\mu \nu}-2 \pi \delta T_{\mu \nu}\right] \xi^{\mu} d \Sigma^{\nu} .
$$

In this equation, $\delta E_{\mu \nu}$ is the linearized equation of motion for a general theory of gravity, and the point-wise equation of motion follows from considering all surfaces $\Sigma$.

In a companion work, one of us [26] shows the relationship of the Wald-Iyer formalism to the present work, where an integral over a surface $\tilde{B}$ rather than a time slice $\Sigma$ appears. Applying the differential operator $\left(\square_{\mathrm{dS}}+d\right)$ to both sides of (5.1) and using the intertwining relation of appendix B along with the equation of motion (4.5) yields the equation [26]

$$
0=\left(\square_{\mathrm{dS}}+d\right) \delta S=R\left[\frac{1}{4 G_{N}} \delta E_{00}-2 \pi \delta T_{00}\right] .
$$

Applying $\left(\square_{\mathcal{K}}+2 d\right)$ instead yields the equation

$$
0=\left(\square_{\mathcal{K}}+2 d\right) \delta S=-R_{\perp}\left[\frac{1}{4 G_{N}} \delta E_{\mu \nu}-2 \pi \delta T_{\mu \nu}\right] .
$$

Hence, the equation of motion for $\delta S$ is equivalent to the linearized gravity equation integrated over a bulk surface, and both vanish due to the entanglement first law.

The localization of the equation of motion onto the Ryu-Takayanagi surface after applying these differential operators, a somewhat surprising fact from the Wald-Iyer point of view, was required by the kinematic space formalism. It would be interesting to study whether such a localization occurs more generally away from the vacuum. If so, it may be more natural to consider whether the bulk gravitational equations can be derived not from entanglement equations, but from one-point entropy equations [27-29]. The Radon transform of the energy density in eq. (3.12) also appears in [30] in the context of relative entropy. There, it was valid in the limit of small CFT regions and low energy excitations of the vacuum. It would be interesting to understand the connection with this work.

It is also possible to assume both the Ryu-Takayanagi formula and Einstein equations, and then derive the kinematic space entropy equations $[11,12]$. One can understand this as a consistency check of the approach, as the entropy equations are pre-determined by conformal invariance.

The techniques we used to derive the quantum corrections for holographic entanglement entropy link this story with a more general program of including interactions in the dynamics of both kinematic space and local bulk operators operators [31-34]. In particular, we can think of $\delta A$ as a kinematic field, whose interactions with the stress tensor generate quantum corrections to the kinematic operator. We will report on interacting kinematic operators in upcoming work. 


\section{Acknowledgments}

We thank Jan de Boer, Michal Heller, Rob Myers, Tadashi Takayanagi, and Sandeep Trivedi for useful conversations. BC thanks the organizers of the workshop "Quantum Information in String Theory and Many-body Systems" at YITP (Kyoto). BC, LL, SM thank the Institute for Advanced Study at Tsinghua University (Beijing) for hospitality during the workshop "An Entangled Trio: Gravity, Information and Condensed Matter." BM is supported by The Netherlands Organisation for Scientific Research (NWO).

\section{A Tensor Radon transforms}

\section{A.1 Tensor intertwinement}

The Radon transform maps functions on AdS to functions on the kinematic space $\mathcal{K}$. In [2] we derived an intertwining relation for the Radon transform of functions, relating the Laplacian on AdS-spacetime to the Laplacian on kinematic space:

$$
\square_{\mathcal{K}} R[f]=-R\left[\square_{\mathrm{AdS}} f\right] .
$$

In this section, we extend the intertwinement relation (A.1) to symmetric two-tensors. We will present a short group theoretic derivation.

The only property of the tensor transform that we will use is that it transforms as a scalar field on kinematic space under the relevant isometry group. Hence, our proof will hold for a general transform $\tilde{R}$ with this transformation property. In particular, $\tilde{R}$ can denote the longitudinal transform $R_{\|}$, the transverse transform $R_{\perp}$, or even the wedge transform used in appendix B.

Consider a tensor transform $\tilde{R}[s]$ of a symmetric 2-tensor field $s_{\mu \nu}$. Under some isometry $g \in \mathrm{SO}(d, 2)$ of $\mathrm{AdS}_{d+1}$, this field transforms as $s \rightarrow g^{\mathrm{AdS}} s$, while a point in kinematic space transforms as $\tilde{B} \rightarrow g \cdot \mathcal{K}$. The scalar transformation property then implies that

$$
\tilde{R}\left[g^{\mathrm{AdS}} s_{\mu \nu}\right](\tilde{B})=\tilde{R}\left[s_{\mu \nu}\right]\left(g^{-1} \stackrel{\mathcal{K}}{B}\right) .
$$

In infinitesimal form equation (A.2) becomes

$$
L_{A B}^{(\mathcal{K}, \text { scalar })} \tilde{R}\left[s_{\mu \nu}\right]=-\tilde{R}\left[L_{A B}^{(\text {AdS }, 2 \text {-tensor })} s_{\mu \nu}\right]
$$

where the $L_{A B}$ are the differential operators representing the generators of the conformal algebra, and the superscript denotes the representation. It follows that

$$
L_{(\mathcal{K}, \text { scalar })}^{2} \tilde{R}[s]=\tilde{R}\left[L_{(\text {AdS }, 2 \text {-tensor })}^{2} s_{\mu \nu}\right],
$$

where $L^{2}=L_{A B} L^{A B}$ is the conformal Casimir.

To find the intertwining relation for the Laplacian, we must now find the quadratic differential operator representing $L^{2}$ on the tensor $s_{\mu \nu}$ and its transform $\tilde{R}[s]$. To do this, 
we make use of the fact that AdS and $\mathcal{K}$ are both coset spaces $G / H$, where $\mathrm{G}=\mathrm{SO}(d, 2)$ and $\mathrm{H}$ is the stabilizer of a bulk point or bulk surface respectively:

$$
\operatorname{AdS}_{d+1}=\frac{\mathrm{SO}(d, 2)}{\mathrm{SO}(d, 1)} ; \quad \mathcal{K}=\frac{\mathrm{SO}(d, 2)}{\mathrm{SO}(d-1,1) \times \mathrm{SO}(1,1)}
$$

The Casimir operator on $\mathrm{G}$ is represented by the Laplacian with respect to the CartanKilling metric. For a coset space $\mathrm{G} / \mathrm{H}$, the Laplacian $\square_{\mathrm{G}}$ can be written as [35]:

$$
\square_{\mathrm{G}}=\square_{\mathrm{G} / \mathrm{H}}+\square_{\mathrm{H}}
$$

A scalar function on AdS-spacetime is in the kernel of the Casimir of the little group $\mathrm{SO}(d, 1)$, so the conformal Casimir is represented on functions on AdS-spacetime by the AdS-Laplacian, up to a constant proportionality factor. A similar argument holds for functions on kinematic space. The relative proportionality factor in the intertwining relation (A.1) is fixed by a choice of the Cartan Killing form on the Lie-algebra of the conformal group $G=\mathrm{SO}(d, 2){ }^{3}$

For general tensors on AdS-spacetime, there will be an additional term from the nontrivial representation of the little group $\mathrm{H}=\mathrm{SO}(d-1,1)$. The tensor Radon transform maps symmetric (two-) tensors on AdS-spacetime to functions on kinematic space, so there will be no additional contributions from the Casimir of the kinematic space little group. Tensors on AdS-spacetime do receive a contribution from the Casimir of the little group $\mathrm{H}=\mathrm{SO}(d, 1)$. One can decompose a general tensor on AdS-spacetime in terms of irreducible representations of the little group $\mathrm{H}=\mathrm{SO}(d-1,1)$. The irreducible representations can be labeled by the spin $\ell$, and the conformal Casimir is represented by [35, 36]:

$$
L_{(\mathrm{AdS}, \ell)}^{2}=-\left(\nabla^{2}+\ell(\ell+d-1)\right) .
$$

where $\nabla^{2}$ denotes the covariant Laplacian. We recover the representation of the conformal Casimir on functions on AdS-spacetime by setting $\ell=0$. The traceless part of a symmetric two-tensor corresponds to the $\ell=2$ representation, whereas the trace-part of a tensor corresponds to the $\ell=0$ representation. We decompose a general symmetric two-tensor $s_{\mu \nu}$ into the traceless symmetric and trace parts

$$
s_{\mu \nu}=s_{\mu \nu}^{\text {trace }}+s_{\mu \nu}^{\text {traceless }}, \quad s_{\mu \nu}^{\text {trace }} \equiv \frac{\operatorname{tr} s}{d+1} g_{\mu \nu} .
$$

Then, using eqs. (A.4), (A.7), and (A.8), we find the following intertwinement rule for symmetric two-tensors:

$$
\begin{aligned}
\square_{K} \tilde{R}\left[s_{\mu \nu}\right] & =-\tilde{R}\left[\nabla^{2} s_{\mu \nu}^{\text {trace }}+\left(\nabla^{2}+2(d+1)\right) s_{\mu \nu}^{\text {traceless }}\right] \\
& =-\tilde{R}\left[\left(\nabla^{2}+2(d+1)\right) s-2 g_{\mu \nu} \operatorname{tr} s\right] .
\end{aligned}
$$

\footnotetext{
${ }^{3}$ For details on the factor of proportionality, see [2].
} 


\section{A.2 Einstein equations from intertwinement}

We would now like to verify eq. (3.9). First recall from eq. (3.7) that the area perturbation can be written as

$$
\delta A=\frac{1}{2} R_{\|}\left[\delta g_{\mu \nu}\right]
$$

From here, we can see that the longitudinal Radon transform annihilates total derivatives:

$$
R_{\|}\left[\nabla_{\mu} v_{\nu}\right]=0
$$

where $v_{\nu}$ is a vector field falling off sufficiently quickly at infinity that the longitudinal transform is well defined. This follows from the fact that $\delta A$ vanishes at first order for small deformations of the surface, which correspond to small coordinate transformations $\delta g_{\mu \nu}=\nabla_{(\mu} v_{\nu)}$.

Applying the Laplacian $\square_{K}$ to $\delta A$ and using the intertwining relation (A.9), we find

$$
\square_{K} \delta A=-\frac{1}{2} R_{\|}\left[\left(\nabla^{2}+2(d+1)\right) \delta g_{\mu \nu}-2 g_{\mu \nu} \operatorname{tr} \delta g\right]
$$

Now, note that the variation of the Ricci tensor is given by

$$
\delta R_{\mu \nu}=\frac{1}{2}\left[\nabla^{\alpha} \nabla_{\mu} \delta g_{\nu \alpha}+\nabla^{\alpha} \nabla_{\nu} \delta g_{\mu \alpha} \quad-\nabla^{2} \delta g_{\mu \nu}-\nabla_{\mu} \nabla_{\nu} \operatorname{tr} \delta g\right]
$$

By commuting covariant derivatives, this can be rewritten for $\mathrm{AdS}_{d+1}$ as

$$
\begin{aligned}
\delta R_{\mu \nu}=\frac{1}{2}[ & \nabla_{\mu}\left(g^{\alpha \beta} \nabla_{\alpha} \delta g_{\nu \beta}\right)+\nabla_{\nu}\left(g^{\alpha \beta} \nabla_{\alpha} \delta g_{\mu \beta}\right) \\
& \left.-\nabla_{\mu} \nabla_{\nu} \operatorname{tr} \delta g-\left(\nabla^{2}+2(d+1)\right) \delta g_{\mu \nu}+2 g_{\mu \nu} \operatorname{tr} \delta g\right] .
\end{aligned}
$$

The last three terms match those in (A.12), while the longitudinal transform annihilate the first three terms, yielding the desired result

$$
\square_{K} \delta A=R_{\|} \delta R_{\mu \nu}
$$

Let us now proceed to verify eq. (3.11). The Hamiltonian constraint equation, the tt component of the Einstein equation, can be written as

$$
R^{\prime}-\operatorname{tr}\left(K^{2}\right)+(\operatorname{tr} K)^{2}=16 \pi G_{N} T_{00}
$$

where $R^{\prime}$ and $K_{\mu \nu}$ denote the Ricci scalar and extrinsic curvature tensors on the time-slice of interest. For an equal-time slice of AdS, we have $K_{\mu \nu}=0$, so that the linearized equation takes the simple form

$$
\delta R^{\prime}=16 \pi G_{N} \delta T_{00} .
$$

Hence, we can prove (3.11) by showing

$$
\left(\square_{\mathrm{dS}}+d\right) \delta A=\frac{1}{2} R\left[\delta R^{\prime}\right] .
$$


If we denote the perturbation of the induced metric on the time-slice of interest by $\delta w_{\mu \nu}$, then we can write $\delta A=\frac{1}{2} R_{\|}\left[\delta w_{\mu \nu}\right]$. Then, both sides of eq. (A.18) depend only on $\delta w_{\mu \nu}$, and we can restrict ourselves to considering perturbations of the metric of hyperbolic space $\mathbb{H}_{d}$. Generalizing (A.9) to hyperbolic space, we can write the intertwining relation

$$
\square_{\mathrm{dS}} R_{\|}\left[\delta w_{\mu \nu}\right]=-R_{\|}\left(\left(\nabla^{2}+2 d\right) \delta w_{\mu \nu}-2 g_{\mu \nu} \operatorname{tr} \delta w\right) .
$$

Using the same methods as above, we find that

$$
\left(\square_{\mathrm{dS}}+d\right) \delta A-\frac{1}{2} R\left[\delta R^{\prime}\right] \propto \int_{\tilde{B}} n^{\mu} n^{\nu} \nabla^{\alpha} \nabla_{[\nu} \delta w_{\alpha] \mu}
$$

where $n^{\mu}$ denotes the unit normal vector to $\tilde{B}$ in $\mathbb{H}_{d}$. To proceed, we must use the fact that the extrinsic curvature tensor on $\tilde{B}$ is zero, which implies that $\nabla_{\mu} n_{\nu}=n_{\mu} n^{\alpha} \nabla_{\alpha} n_{\nu}$. Repeatedly using this along with eq. (A.11), we find that the right-hand side of (A.20) vanishes, proving the result (3.11).

\section{B Wedge integral relations}

In this appendix, we will prove that the equation (4.10) is satisfied by the bulk modular Hamiltonian (4.12). The object of interest will be a transformation $R_{\wedge}\left[s_{\mu \nu}\right]$ of a conserved symmetric 2-tensor $s_{\mu \nu}$, defined by

$$
R_{\wedge}\left[s_{\mu \nu}\right]=\int_{\Sigma} s_{\mu \nu} \xi^{\mu} d \Sigma^{\nu}
$$

where $\Sigma$ is an equal-time slice of a causal wedge, $d \Sigma^{\nu}$ is the unit normal vector to that slice, and $\xi^{\mu}$ is a Killing vector given in Poincaré coordinates by [16]

$$
\xi^{\mu}=\frac{\left(X-X_{1}\right)^{2}\left(X-X_{2}\right)^{\mu}-\left(X-X_{2}\right)^{2}\left(X-X_{1}\right)^{\mu}}{\left(X_{2}-X_{1}\right)^{2}}
$$

Here the capital letters denote bulk coordinates $X=(x, z)$, whose indices are contracted using the Minkowski metric. The bottom and top points of the corresponding causal diamond are denoted by $X_{1}=\left(x_{1}, 0\right)$ and $X_{2}=\left(x_{2}, 0\right)$ respectively, with $\xi$ representing a flow from $X_{2}$ to $X_{1}$. In particular, we have $H_{\text {bulk }}=2 \pi R_{\wedge}\left[T_{\mu \nu}\right]$. Note that since $s_{\mu \nu}$ is conserved, $R_{\wedge}$ is independent the choice of time slice $\Sigma$.

We can now restrict ourselves to a constant time slice of $\mathrm{AdS}_{d+1}$, which we take to be the $t=0$ slice. Then, $\xi$ points only in the time direction, and we have

$$
R_{\wedge}\left[s_{\mu \nu}\right]=\int_{\Sigma} s_{00}|\xi| d \Sigma .
$$

Hence, this restriction of the wedge transform is really a scalar transform, and is subject to the scalar intertwining relation considered in [2]. In particular, we have

$$
\left(\square_{\mathrm{dS}}+d\right) R_{\wedge}\left[s_{\mu \nu}\right]=\int_{\Sigma}\left(-\square_{\mathbb{H}}+d\right) s_{00}|\xi|
$$


Integrating by parts, this becomes

$$
\left(\square_{\mathrm{dS}}+d\right) R_{\wedge}\left[s_{\mu \nu}\right]=-\int_{\partial \Sigma}|\xi| n^{\mu} \nabla_{\mu} s_{00}+\int_{\Sigma} s_{00}\left(d-\nabla^{2}\right)|\xi|+\int_{\partial \Sigma} s_{00} n^{\mu} \nabla_{\mu}|\xi|
$$

where $n^{\mu}$ is the outward-pointing unit normal vector to $\tilde{B}$ within the specified time slice. The first term vanishes because $|\xi|=0$ at $\tilde{B}$, and because $s_{00}$ goes to zero sufficiently quickly at $\partial \mathrm{AdS}$; the second vanishes since $\nabla_{\mathbb{H}}^{2}|\xi|=d|\xi|$, as can be checked explicitly from (B.2). Finally, we can check from (B.2) that $n^{\mu} \nabla_{\mu}|\xi|=-1$. This gives us our result,

$$
\left(\square_{\mathrm{dS}}+d\right) R_{\wedge}\left[s_{\mu \nu}\right]=-R\left[s_{\mu \nu} \hat{t}^{\mu} \hat{t}^{\nu}\right]
$$

where $\hat{t}$ is the timelike unit vector to the time slice correponding to the chosen de Sitter slice of kinematic space.

With some more effort, we can also prove a similar relation using the Laplacian on the full kinematic space, rather than a de Sitter slice. First, note that the wedge integral can be written as

$$
R_{\wedge}\left[s_{\mu \nu}\right]=\int_{\Sigma} * j .
$$

where we have defined the conserved current

$$
j_{\mu}=s_{\mu \nu} \xi^{\nu}
$$

Now, since $R_{\wedge}$ transforms as a scalar in the full kinematic space, we can use the tensor intertwining relation (A.9) to obtain

$$
\begin{aligned}
\left(\square_{K}+2 d\right) R_{\wedge}\left[s_{\mu \nu}\right] & =R_{\wedge}\left[-\left(\nabla^{2}+2\right) s_{\mu \nu}+2 g_{\mu \nu} \operatorname{tr} s\right] \\
& =\int_{\Sigma} * \tilde{j}
\end{aligned}
$$

where $\tilde{j}_{\mu}=\left[-\left(\nabla^{2}+2\right) s_{\mu \nu}+2 g_{\mu \nu} \operatorname{tr} s\right] \xi^{\nu}$. Using the fact that $s_{\mu \nu}$ is conserved and $\xi^{\mu}$ is Killing, it can be shown with significant effort [26] that $\tilde{j}=\Delta j$, where $\Delta$ is the Hodge Laplacian. Then, conservation of $j$ implies that

$$
\begin{aligned}
\left(\square_{K}+2 d\right) R_{\wedge}\left[s_{\mu \nu}\right] & =-\int_{\tilde{B}} * d j . \\
& =-\frac{1}{2} \int_{\tilde{B}} \epsilon^{\mu \nu}(d j)_{\mu \nu}
\end{aligned}
$$

where $\epsilon^{\mu \nu}$ is the antisymmetric tensor in the two directions perpendicular to $\partial \Sigma$, defined such that $\epsilon^{01}=-1$. Next, note that since $\xi$ vanishes on $\tilde{B}$, we can plug in $j$ to find

$$
\left(\square_{K}+2 d\right) R_{\wedge}\left[s_{\mu \nu}\right]=\int_{\tilde{B}}\left(\epsilon^{\mu \alpha} \nabla_{\alpha} \xi^{\nu}\right) s_{\mu \nu} .
$$

Finally, it can be checked explicitly from (B.2) that $\epsilon^{\mu \alpha} \nabla_{\alpha} \xi^{\nu}=g^{\mu \nu}-h^{\mu \nu}$, where $h^{\mu \nu}$ is the induced metric on $\tilde{B}$. This yields the result

$$
\left(\square_{K}+2 d\right) R_{\wedge}\left[s_{\mu \nu}\right]=R_{\perp}\left[s_{\mu \nu}\right] .
$$

This relation was required for consistency between equations (3.10), (4.7), and (4.13). 


\section{Modular Hamiltonian as an OPE block}

In this appendix, we will relate the stress tensor OPE block to the vacuum modular Hamiltonian for a spherical CFT region. This implies that the vacuum modular Hamiltonian appears as the contribution of the stress tensor to the OPE of timelike separated scalars of equal dimension, or the expansion of a spherical operator as in eq. (2.5).

In the OPE of two timelike separated scalars $O(x)$ of equal scaling dimension $\Delta$, the stress tensor and its derivatives appear as

$$
\frac{O\left(x_{1}\right) O\left(x_{2}\right)}{\left\langle O\left(x_{1}\right) O\left(x_{2}\right)\right\rangle} \supset C_{O O T} \underbrace{(1+\ldots) \frac{x_{12}^{\mu} x_{12}^{\nu}}{x_{12}^{2-d}} T_{\mu \nu}\left(\frac{x_{1}+x_{2}}{2}\right)}_{\mathcal{B}_{T}\left(x_{1}, x_{2}\right)} .
$$

Here, we have defined the OPE block $\mathcal{B}_{T}$ for the stress tensor, which includes the contribution of $T_{\mu \nu}$ and all descendants to the OPE, and is independent of the choice of operator $O(x)$. Note that, when expanded about the point $\frac{x_{1}+x_{2}}{2}$, we can use tracelessness and conservation to ensure that only the quantity $x_{12}^{\mu} x_{12}^{\nu} T_{\mu \nu}$ and its derivatives in directions perpendicular to $x_{12}$ appear. More concretely, if we choose $x_{2}=-x_{1}=R \hat{t}$, this means that only $T_{00}$ and its spatial derivatives appear, as we would expect from the expression (4.4).

Now, consider the $\mathrm{SO}(d, 1)$ subgroup of the conformal group which preserves the time slice intersecting $\frac{x_{1}+x_{2}}{2}$ and the sphere corresponding to $x_{1}, x_{2}$. The quantity $x_{12}^{\mu} x_{12}^{\nu} T_{\mu \nu}$ transforms as a scalar primary of dimension $d$ under this subgroup, so it has eingenvalue $-\Delta(\Delta-(d-1))=-d$ under the Casimir operator $L_{\mathrm{SO}(d, 1)}^{2}$, as do its derivatives in directions along the time slice. Since $\mathcal{B}_{T}\left(x_{1}, x_{2}\right)$ transforms as a scalar in kinematic space, $L_{\mathrm{SO}(d, 1)}^{2}$ is represented by $\square_{\mathrm{dS}}$, the Laplacian on the de Sitter slice of kinematic space corresponding to this $\mathrm{SO}(d, 1)$ subgroup [14]. Hence, $\mathcal{B}_{T}$ obeys the equation of motion

$$
\left(\square_{\mathrm{dS}}+d\right) \mathcal{B}_{T}=0
$$

which of course matches eq. (4.5) for the modular Hamiltonian; in fact, we obtain a whole family of such equations, one for each time slice. Comparing boundary conditions of (4.8) and (C.1), we obtain the relation

$$
\mathcal{B}_{T}=-\frac{2^{d}\left(d^{2}-1\right)}{2 \pi \Omega_{d-2}} H_{\text {mod }}
$$

where $\Omega_{d-2}$ is the area of a $(d-2)$-sphere. This result can also be obtained through the shadow operator formalism [2].

Open Access. This article is distributed under the terms of the Creative Commons Attribution License (CC-BY 4.0), which permits any use, distribution and reproduction in any medium, provided the original author(s) and source are credited.

\section{References}

[1] B. Czech, L. Lamprou, S. McCandlish and J. Sully, Integral geometry and holography, JHEP 10 (2015) 175 [arXiv:1505.05515] [INSPIRE]. 
[2] B. Czech, L. Lamprou, S. McCandlish, B. Mosk and J. Sully, A stereoscopic look into the bulk, JHEP 07 (2016) 129 [arXiv: 1604.03110] [INSPIRE].

[3] Virgo and LigO Scientific collaborations, B.P. Abbott et al., Observation of gravitational waves from a binary black hole merger, Phys. Rev. Lett. 116 (2016) 061102 [arXiv: 1602.03837] [INSPIRE].

[4] L. Susskind, The world as a hologram, J. Math. Phys. 36 (1995) 6377 [hep-th/9409089] [INSPIRE].

[5] G. 't Hooft, Dimensional reduction in quantum gravity, gr-qc/9310026 [INSPIRE].

[6] S. Ryu and T. Takayanagi, Holographic derivation of entanglement entropy from AdS/CFT, Phys. Rev. Lett. 96 (2006) 181602 [hep-th/0603001] [INSPIRE].

[7] V.E. Hubeny, M. Rangamani and T. Takayanagi, A covariant holographic entanglement entropy proposal, JHEP 07 (2007) 062 [arXiv:0705.0016] [INSPIRE].

[8] N. Lashkari, M.B. McDermott and M. Van Raamsdonk, Gravitational dynamics from entanglement 'thermodynamics', JHEP 04 (2014) 195 [arXiv: 1308.3716] [INSPIRE].

[9] T. Faulkner, M. Guica, T. Hartman, R.C. Myers and M. Van Raamsdonk, Gravitation from entanglement in holographic CFTs, JHEP 03 (2014) 051 [arXiv:1312.7856] [INSPIRE].

[10] B. Swingle and M. Van Raamsdonk, Universality of gravity from entanglement, arXiv: 1405.2933 [INSPIRE].

[11] M. Nozaki, T. Numasawa, A. Prudenziati and T. Takayanagi, Dynamics of entanglement entropy from Einstein equation, Phys. Rev. D 88 (2013) 026012 [arXiv:1304.7100] [INSPIRE].

[12] J. Bhattacharya and T. Takayanagi, Entropic counterpart of perturbative Einstein equation, JHEP 10 (2013) 219 [arXiv: 1308.3792] [INSPIRE].

[13] G. Wong, I. Klich, L.A. Pando Zayas and D. Vaman, Entanglement temperature and entanglement entropy of excited states, JHEP 12 (2013) 020 [arXiv: 1305.3291] [INSPIRE].

[14] J. de Boer, M.P. Heller, R.C. Myers and Y. Neiman, Holographic de Sitter geometry from entanglement in conformal field theory, Phys. Rev. Lett. 116 (2016) 061602 [arXiv: 1509.00113] [INSPIRE].

[15] D. Allahbakhshi, M. Alishahiha and A. Naseh, Entanglement thermodynamics, JHEP 08 (2013) 102 [arXiv: 1305. 2728] [INSPIRE].

[16] J. de Boer, F.M. Haehl, M.P. Heller and R.C. Myers, Entanglement, holography and causal diamonds, JHEP 08 (2016) 162 [arXiv:1606.03307] [INSPIRE].

[17] G. Solanes, Integral geometry and curvature integrals in hyperbolic space, Universitat Autonoma de Barcelona, Barcelona Spain, (2003).

[18] T. Faulkner, A. Lewkowycz and J. Maldacena, Quantum corrections to holographic entanglement entropy, JHEP 11 (2013) 074 [arXiv: 1307.2892] [INSPIRE].

[19] S. Helgason, The Radon transform, volume 5, Springer, U.S.A., (1999).

[20] V. Balasubramanian and P. Kraus, A stress tensor for anti-de Sitter gravity, Commun. Math. Phys. 208 (1999) 413 [hep-th/9902121] [INSPIRE].

[21] R.C. Myers, Stress tensors and Casimir energies in the AdS/CFT correspondence, Phys. Rev. D 60 (1999) 046002 [hep-th/9903203] [INSPIRE]. 
[22] S. de Haro, S.N. Solodukhin and K. Skenderis, Holographic reconstruction of space-time and renormalization in the AdS/CFT correspondence, Commun. Math. Phys. 217 (2001) 595 [hep-th/0002230] [INSPIRE].

[23] H. Casini, M. Huerta and R.C. Myers, Towards a derivation of holographic entanglement entropy, JHEP 05 (2011) 036 [arXiv:1102.0440] [INSPIRE].

[24] D.L. Jafferis, A. Lewkowycz, J. Maldacena and S.J. Suh, Relative entropy equals bulk relative entropy, JHEP 06 (2016) 004 [arXiv: 1512.06431] [INSPIRE].

[25] V. Iyer and R.M. Wald, A comparison of Noether charge and Euclidean methods for computing the entropy of stationary black holes, Phys. Rev. D 52 (1995) 4430 [gr-qc/9503052] [INSPIRE].

[26] B. Mosk, Holographic equivalence between the first law of entanglement entropy and the linearized gravitational equations, Phys. Rev. D 94 (2016) 126001 [arXiv:1608.06292] [INSPIRE].

[27] V.E. Hubeny and M. Rangamani, Causal holographic information, JHEP 06 (2012) 114 [arXiv: 1204.1698] [INSPIRE].

[28] W.R. Kelly and A.C. Wall, Coarse-grained entropy and causal holographic information in AdS/CFT, JHEP 03 (2014) 118 [arXiv:1309.3610] [INSPIRE].

[29] W.R. Kelly, Deriving the first law of black hole thermodynamics without entanglement, JHEP 10 (2014) 192 [arXiv: 1408.3705] [INSPIRE].

[30] J. Lin, M. Marcolli, H. Ooguri and B. Stoica, Locality of gravitational systems from entanglement of conformal field theories, Phys. Rev. Lett. 114 (2015) 221601 [arXiv: 1412.1879] [INSPIRE].

[31] D. Kabat, G. Lifschytz and D.A. Lowe, Constructing local bulk observables in interacting AdS/CFT, Phys. Rev. D 83 (2011) 106009 [arXiv:1102.2910] [InSPIRE].

[32] D. Kabat and G. Lifschytz, Bulk equations of motion from CFT correlators, JHEP 09 (2015) 059 [arXiv: 1505. 03755] [INSPIRE].

[33] D. Kabat and G. Lifschytz, Locality, bulk equations of motion and the conformal bootstrap, JHEP 10 (2016) 091 [arXiv:1603.06800] [INSPIRE].

[34] I. Heemskerk, D. Marolf, J. Polchinski and J. Sully, Bulk and transhorizon measurements in AdS/CFT, JHEP 10 (2012) 165 [arXiv:1201.3664] [INSPIRE].

[35] K. Pilch and A.N. Schellekens, Formulae for the eigenvalues of the Laplacian on tensor harmonics on symmetric coset spaces, J. Math. Phys. 25 (1984) 3455 [INSPIRE].

[36] E. Hijano, P. Kraus, E. Perlmutter and R. Snively, Witten diagrams revisited: the AdS geometry of conformal blocks, JHEP 01 (2016) 146 [arXiv:1508.00501] [INSPIRE]. 